\title{
BMJ Open Effect of routinely assessing and addressing depression and diabetes distress using patient-reported outcome measures in improving outcomes among adults with type 2 diabetes: a systematic review protocol
}

Rita McMorrow (D) ,' Barbara Hunter (D) , ${ }^{1}$ Christel Hendrieckx, ${ }^{2,3}$ Dominika Kwasnicka, ${ }^{4,5}$ Leanne Cussen, ${ }^{6}$ Felicia Ching Siew Ho, ${ }^{7}$ Jane Speight, ${ }^{2,3}$ Jon Emery (D) , ${ }^{8}$ Jo-Anne Manski-Nankervis (iD) ${ }^{1}$

To cite: McMorrow $\mathrm{R}$, Hunter B, Hendrieckx C, et al. Effect of routinely assessing and addressing depression and diabetes distress using patient-reported outcome measures in improving outcomes among adults with type 2 diabetes: a systematic review protocol. BMJ Open 2021;11:e044888. doi:10.1136/ bmjopen-2020-044888

- Prepublication history and supplemental material for this paper is available online. To view these files, please visit the journal online (http://dx.doi. org/10.1136/bmjopen-2020044888).

Received 16 September 2020 Revised 05 February 2021 Accepted 18 February 2021

Check for updates

(c) Author(s) (or their employer(s)) 2021. Re-use permitted under CC BY-NC. No commercial re-use. See rights and permissions. Published by BMJ.

For numbered affiliations see end of article.

Correspondence to

Dr Rita McMorrow;

rita.mcmorrow@unimelb.edu.au

\section{ABSTRACT}

Introduction Type 2 diabetes is a global health priority. People with diabetes are more likely to experience mental health problems relative to people without diabetes. Diabetes guidelines recommend assessment of depression and diabetes distress during diabetes care. This systematic review will examine the effect of routinely assessing and addressing depression and diabetes distress using patient-reported outcome measures in improving outcomes among adults with type 2 diabetes.

Methods and analysis MEDLINE, Embase, CINAHL Complete, PsycInfo, The Cochrane Library and Cochrane Central Register of Controlled Trials will be searched using a prespecified strategy using a prespecified Population, Intervention, Comparator, Outcomes, Setting and study design strategy. The date range of the search of all databases will be from inception to 3 August 2020. Randomised controlled trials, interrupted time-series studies, prospective and retrospective cohort studies, case-control studies and analytical cross-sectional studies published in peer-reviewed journals in the English language will be included. Two review authors will independently screen abstracts and full texts with disagreements resolved by a third reviewer, if required, using Covidence software. Two reviewers will undertake risk of bias assessment using checklists appropriate to study design. Data will be extracted using prespecified template. A narrative synthesis will be conducted, with a meta-analysis, if appropriate.

Ethics and dissemination Ethics approval is not required for this review of published studies. Presentation of results will follow the Preferred Reporting Items for Systematic reviews and MetaAnalyses guidance. Findings will be disseminated via peer-reviewed publication and conference presentations.

PROSPERO registration number CRD42020200246.
Strengths and limitations of this study

- This systematic review will examine the effect of routinely assessing and addressing depression and diabetes distress in improving the outcomes of type 2 diabetes as part of routine care, which is recommended in clinical diabetes guidelines.

- Our review will assess the impact of patientreported outcome use in type 2 diabetes on a range of clinical outcomes including glycaemia, depressive symptoms, diabetes distress, well-being and diabetes self-management.

- The literature is expected to be heterogeneous in terms of the patient-reported outcome measures used for depression and diabetes distress and may need to be reported as a narrative synthesis.

\section{INTRODUCTION}

\section{Rationale}

Type 2 diabetes (T2D) is a global health priority with an estimated 463 million people living with diabetes in 2017, set to rise to 700 million people in 2045. ${ }^{1}$ T2D impacts not only on physical health but also on mental health. Up to 4 in 10 people with T2D experience problems related to mental health, such as depression, anxiety and diabetes distress. ${ }^{2}$ Diabetes distress, the negative psychological reaction to the emotional burden of living with and managing diabetes, is experienced by up to $36 \%$ of people with T2D. ${ }^{34}$ Depression and diabetes can coexist. Diabetes distress is not a diagnosable mental health issue but an emotional response related to the day-to-day living with diabetes. ${ }^{56}$ Impaired mental health is associated with reduced self-management and increased risk of suboptimal glycaemia, 
diabetes-related complications, impaired quality of life, mortality and an estimated $50 \%$ increase in healthcare costs. $^{7-13}$

Diabetes guidelines have acknowledged the importance of psychological assessment as part of diabetes care for over 25 years. ${ }^{14}$ The International Diabetes Federation recommends screening for depression with a validated tool in primary care diabetes clinics and referring those who screen positively to a mental healthcare professional with expertise in diabetes. ${ }^{15}$ The American College for Endocrinology, The National Institute for Healthcare and Excellence in the UK, Royal Australian College of General Practitioners, American Diabetes Association and Diabetes Canada all have similar recommendations for routine psychological assessment as part of diabetes care. ${ }^{15-20}$ Across these guidelines, there is considerable variation in terms of which patient-reported outcome measures (PROMs) are recommended. PROMs are standardised, validated questionnaires that are completed by patients to assess latent constructs such as emotional well-being, treatment satisfaction, perceived health or functional status or health-related quality of life. ${ }^{21}$ Recent consensus from the International Consortium of Health Outcomes Measurement recommends annual psychological assessment as part of diabetes care using the Problem Areas in Diabetes scale (PAID), WHO-Five Well-Being Index (WHO-5) and Patient Health Questionnaire-9 (PHQ-9). ${ }^{22}$

Despite these long-standing recommendations for PROM use as a component of the assessment of depression and diabetes distress as part of diabetes care and the known consequences of impaired mental health, less than one-third of people with diabetes recall healthcare professionals asking about anxiety or depression symptoms. ${ }^{23}$ While use of PROMs enables people with diabetes to self-reflect on their condition with increased patient concerns being discussed with healthcare professionals, ${ }^{24}$ healthcare professionals report low rates of training in care for people with mental health issues related to diabetes, with two-thirds identifying the need for training in managing the psychological aspects of diabetes care. ${ }^{25}$ Barriers to the assessment of diabetes distress reported by healthcare professionals include lack of confidence when addressing mental health as part of diabetes care. ${ }^{26}$ Recent systematic reviews have focused on the role and benefits of interventions for the management of diabetes distress; however, the first step in delivering psychological care is to identify those who require such interventions. ${ }^{27-29}$ With the rising prevalence of T2D worldwide, the majority of whom are managed in general practice, and specialist psychological support with expertise in diabetes care being scarce, there is an urgent need to ensure integrated mental health assessment to enable holistic diabetes care. ${ }^{16} \mathrm{We}$ need to develop an efficient system to assess for diabetes distress as part of routine diabetes care provided in general practice. ${ }^{6}$

\section{The current study}

For PROMs to be successfully implemented into routine diabetes care amidst increasing pressures on clinical time (particularly in primary care), clinicians need to understand the utility of the tools to support the patient and improve clinical outcomes, not just for audit or research purposes. ${ }^{3031}$ To engage healthcare professionals in the use of PROMs for the assessment of depression and diabetes distress, we must understand the impact that performing these assessments in routine diabetes care has on clinical, process of care and patient-reported outcomes. Impact may be mediated by the method of completion (eg, online or paper-based assessment), the type of healthcare professional and their setting (eg, primary vs specialist care). The resulting insights will provide a foundation for further translation of the guidelines recommending mental health assessment as part of routine diabetes care.

\section{Objectives}

This review will examine the effect of routinely assessing and addressing depression and diabetes distress on clinical and patient-reported outcomes among adults with T2D.

\section{METHODS AND ANALYSIS}

This protocol was prepared using the Preferred Reporting Items for Systematic Reviews and Meta-Analyses Protocols (PRISMA-P) guidelines. ${ }^{32}$

\section{Patient and public involvement}

This protocol was discussed with members of our consumer advisory group. The aims, methods and type of healthcare professionals who received the feedback of the PROM were discussed. The consumer advisory group agreed with the aims and methods of the review. Several members indicated the importance of including studies involving any healthcare professional, not just doctors and nurses, in the review.

\section{Eligibility criteria}

\section{Study characteristics}

Randomised controlled trials, interrupted time-series studies, prospective and retrospective cohort studies, case-control studies and analytical cross-sectional studies published in the English language will be included. Qualitative studies, case studies, animal studies and conference abstracts will be excluded.

\section{Population}

Our target study population is adults (18 years of age or older) with T2D from any country. Studies including children and adolescents (under 18 years of age), people without T2D (eg, type 1 diabetes or gestational diabetes) will be excluded.

\section{Intervention}

The intervention includes both of the following: 
1. Completion of PROMs by an adult with T2D, including self-completed or interviewer-administered measures of:

- Depressive symptoms, for example, $\mathrm{PHQ}^{33}$; the Beck Depression Inventory ${ }^{33}$ or Centre of Epidemiological Studies-Depression. ${ }^{34}$

- Diabetes distress, for example, Problem Areas in Diabetes ${ }^{35}$ or Diabetes Distress Scale (DDS) ${ }^{36}$ The PROMs to be included will be broadened based on the PROMs measuring depressive symptoms or diabetes distress identified during the search.

2. Feeding of PROM responses back to and/or use of PROM responses by the treating healthcare professional in consultation with the person with T2D.

The PROMs to be included will be broadened based on the PROMs measuring depressive symptoms or diabetes distress identified during the search.

\section{Outcomes}

Our primary outcome of interest is glycaemia as measured by glycated haemoglobin (HbA1c).

Secondary outcomes of interest include:

1. Reported depressive symptoms and diabetes distress responses at follow-up.

2. Reported psychological well-being or health-related quality of life at follow-up, for example, the WHO-5, EQ-5D ${ }^{37}$ or SF-36. ${ }^{38}$

3. Reported diabetes self-management at follow-up, for example, change in diabetes self-management as measured by the Summary of Diabetes Self-Care Activities. $^{39}$

4. Number of referrals for psychiatric or psychological assessment or therapy.

5. Reported patient and doctor communication.

6. Reported satisfaction with consultation.

\section{Information sources}

The following databases will be searched: MEDLINE (Ovid), Embase (Ovid), CINAHL Complete (EBSCO), APA PsycInfo (Ovid), The Cochrane Library (Ovid) and Cochrane Central Register of Controlled Trials (Ovid). The search strategy will be specific to MEDLINE (Ovid) but adapted for other databases using the appropriate controlled vocabulary prior to conducting the searches. There will be no restriction on publication date.

\section{Reference lists and correspondence}

We will screen reference lists (of included trial reports and systematic reviews) to identify additional studies and will contact experts in the field for information on unpublished studies, or to request additional trial data. As the terminology of "patient-reported outcome measures' and 'PROMs' has been adopted relatively recently in the literature, we will also search specifically for common validated measures of depressive symptoms or diabetes distress.

\begin{tabular}{|c|c|c|}
\hline Type 2 diabetes & PROMs & Mental health \\
\hline $\begin{array}{l}\text { Diabetes Mellitus, } \\
\text { Type 2/ }\end{array}$ & $\begin{array}{l}\text { Patient Reported } \\
\text { Outcome/ }\end{array}$ & depress* \\
\hline Type 2 Diabetes & $\begin{array}{l}\text { Patient-reported } \\
\text { outcome* }\end{array}$ & distress* \\
\hline Type II Diabetes & PROM & emotion* \\
\hline T2DM & PRO & psycholog* \\
\hline Diabetes Mellitus & K10 & psychosocial \\
\hline \multirow[t]{4}{*}{ NIDDM } & $\mathrm{PHQ}$ & wellbeing \\
\hline & PAID & well-being \\
\hline & DDS & "well being" \\
\hline & & mental \\
\hline
\end{tabular}

*truncation operator.

DDS, Diabetes Distress Scale; NIDDM, non-insulin-dependent diabetes mellitus; PAID, Problem Areas in Diabetes; PHQ, Patient Health Questionnaire; PROM, patient-reported outcome measure; T2DM, type 2 diabetes mellitus.

\section{Search strategy}

The abbreviated search strategy is listed in table 1 . The initial searches will be conducted with MEDLINE, and adjustments will be made before the final search of all databases. The full MEDLINE search is in online supplemental file 1 . The date range of the search of all databases will be from inception to 3 August 2020. The initial search strategy was conducted on 3 August 2020.

\section{Data management}

Search results will be imported to EndNote (V.X9.3.3, Clarivate Analytics) for removal of duplicates. Unique records will be imported to Covidence for the selection and screening process. ${ }^{40}$

\section{Selection process}

All abstracts will be screened by two reviewers independently against the predefined eligibility criteria outlined. Any inter-reviewer disagreements will be discussed and resolved, where necessary, with a third reviewer. Full-text screening of all positively screened abstracts will be conducted by two reviewers independently, with disagreements resolved using the same procedure. Reasons for exclusion of full texts will be recorded using Covidence.

\section{Data extraction process}

Data will be extracted from all included studies and recorded in a data extraction form following a prespecified Participants, Intervention, Comparator, Outcomes framework (see online supplemental file 2). The data extraction form will be refined and adjusted if necessary.

\section{Data to be extracted}

\section{Participants}

$\mathrm{N}$, age (years), gender, socioeconomic status, education level, diabetes duration (years), HbAlc, insulin treatment $(\mathrm{Y} / \mathrm{N})$, comorbidities (number and type), known 
mental health diagnosis, prior referral to psychologist or psychiatrist, baseline PROM scores (including depression and diabetes distress), severe diabetes distress (eg, PAID $>40$, DDS $>18$ ), ratings of doctor-patient communication (if available).

\section{Intervention}

Specific instrument/PROM used, frequency of completion, method of completion, location of completion, feedback of PROM responses to healthcare professional, type of healthcare professional receiving PROM responses, method of feedback, training on interpretation of PROM responses, associated action given to the healthcare professional based on the outcomes, feedback on the PROM responses to the person with diabetes, associated action PROM responses for the person with diabetes, other co-interventions, for example, additional training in motivational interviewing/guidance handbooks/extra psychological support for people with diabetes.

\section{Comparator}

Details of treatment provided to the comparison group.

\section{Outcome}

Primary and secondary outcomes specified and collected, and time points reported.

\section{Study design and setting}

Study design, total duration of study, number of study centres and location, study setting, participants' recruitment, protocol adherence, study drop-out rate.

\section{Outcomes and prioritisation}

If sufficient studies are identified, a meta-analysis will be conducted using RevMan. ${ }^{41}$ For our primary outcome, glycaemia, we will divide studies into groups based on the reporting of HbAlc as a continuous variable or a categorical variable. We will calculate risk ratios with 95\% CIs for dichotomous data.

For continuous outcomes, such as depressive symptoms or diabetes distress, well-being or self-management mean differences (MDs) with 95\% CIs will be used. When studies use different scales or measurements for the same underlying construct, we will use the standardised MD and the associated 95\% CI. For categorical (binary) outcomes, such as referrals for psychological therapy, we will use logistic regression to calculate ORs and the associated $95 \%$ CIs.

\section{Dealing with missing data}

We will contact authors to confirm study characteristics and obtain missing data where possible (for example, when a study has both participants with type 1 diabetes and T2D). We will allow for a maximum of 1 month and three emails for responses. We will document all correspondence and report responses in the final review.

\section{Risk of bias in individual studies}

Two reviewers will independently assess the risk of bias in each of the included studies using the risk of bias for randomised trials and Risk Of Bias In Non-randomised Studies-Interventions for non-randomised studies of interventions, as recommended by the Cochrane Handbook. ${ }^{42-44}$ Any inter-reviewer disagreements about risk of bias will be discussed and resolved with a third reviewer.

\section{Data synthesis}

A meta-analysis will be conducted if appropriate using RevMan, that, is if the interventions, participants, study design, outcomes and number of identified papers are sufficiently homogeneous. Between study statistical heterogeneity will be assessed using the $\mathrm{I}^{2}$ statistic. If sufficient studies, a funnel plot will be created to assess publication bias. If a meta-analysis is not possible, the outcomes will be discussed in a narrative synthesis.

A narrative synthesis and summary of findings will describe the findings from each included study. We will present the following details: the number and characteristics of participants in each study, study setting and design, PROM used, risk of bias of the study, findings for effects on participant outcomes including PROM responses, HbAlc, and on the clinical and process of care outcomes.

\section{Confidence in the cumulative evidence}

As recommended by the Cochrane Handbook, we will use the Grading of Recommendations, Assessment, Development and Evaluation criteria to grade the quality and strength of evidence. ${ }^{45}$

\section{ETHICS AND DISSEMINATION}

Ethics was not required for this study as it does not involve the collection of individual patient data. We will disseminate results via peer-reviewed publication, conference presentations and local networks via newsletters and social media. This systematic review is part of the lead author's (RM) PhD.

\section{DISCUSSION}

This systematic review will synthesise the evidence related to the impact of assessing depression and diabetes distress using PROMs on glycaemia, the wellbeing of people with diabetes and processes of care. People with diabetes are more likely to experience mental health issues; to address this we must understand the most effective ways of assessing depression and diabetes distress in diabetes care. ${ }^{2}$ Our review will make an important contribution to the development of an intervention to allow for assessment of depression and diabetes distress in general practice. Given the majority of people with diabetes attend their general practitioner as part of routine care, we suspect this is the 
appropriate context for these assessments to occur. ${ }^{46}$ As impaired mental health is associated with increased risk of suboptimal glycaemia and self-management strategies, general practice is well positioned to provide holistic care related to both mental health and diabetes self-management. ${ }^{7-9}$

Key strengths of this systematic review will be the adherence to the PRISMA guidelines and an experienced team of reviewers with all screening and data extraction checked independently by two reviewers. A limitation of this review is that there are a wide variety of PROMs used for the assessment of depression and diabetes distress in T2D care and we expect a heterogeneous group of articles, which may result in a meta-analysis potentially not being performed. The restriction of studies to those written in English may also be a limitation.

This review will highlight key areas and most effective ways of conducting assessment of depressive symptoms and diabetes distress in clinical care. Future directions include using the results from this systematic review to guide the development of an intervention to allow for the translation of international guidance for assessment of depressive symptoms and diabetes distress routinely during diabetes care.

\section{Author affiliations}

${ }^{1}$ Department of General Practice, The University of Melbourne, Melbourne, Victoria, Australia

${ }^{2}$ Diabetes Victoria, The Australian Centre for Behavioural Research in Diabetes, Melbourne, Victoria, Australia

${ }^{3}$ School of Psychology, Deakin University, Geelong, Victoria, Australia

${ }^{4}$ NHMRC CRE in Digital Technology to Transform Chronic Disease Outcomes,

Melbourne School of Population and Global Health, The University of Melbourne, Melbourne, Victoria, Australia

${ }^{5}$ Faculty of Psychology, SWPS University of Social Sciences and Humanities, Warsaw, Mazovia, Poland

${ }^{6}$ Department of Endocrinology, Beaumont Hospital, Dublin, Ireland

${ }^{7}$ Melbourne Medical School, The University of Melbourne, Melbourne, Victoria, Australia

${ }^{8}$ Department of General Practice and Centre for Cancer Research, The University of Melbourne, Melbourne, Victoria, Australia

Twitter Jane Speight @janespeight and Jo-Anne Manski-Nankervis @jo_manski

Contributors RM conceived the project and obtained project funding. RM is the project lead and coordinator, and she drafted the manuscript, search strategy and inclusion/exclusion criteria, with conceptual contributions to project design and procedures from J-AM-N, BH, CH, DK, LC FCSH, JS and JE. All authors read, edited and approved the final manuscript.

Funding The Australian Rotary Health and the University of Melbourne supported this study through a PhD scholarship for RM (grant number: N/A). CH and JS are supported by core funding to the Australian Centre for Behavioural Research in Diabetes derived from the collaboration between Diabetes Victoria and Deakin University.

Disclaimer The funding body had no role in developing the protocol.

Competing interests None declared.

Patient consent for publication Not required.

Provenance and peer review Not commissioned; externally peer reviewed.

Supplemental material This content has been supplied by the author(s). It has not been vetted by BMJ Publishing Group Limited (BMJ) and may not have been peer-reviewed. Any opinions or recommendations discussed are solely those of the author(s) and are not endorsed by BMJ. BMJ disclaims all liability and responsibility arising from any reliance placed on the content. Where the content includes any translated material, BMJ does not warrant the accuracy and reliability of the translations (including but not limited to local regulations, clinical guidelines, terminology, drug names and drug dosages), and is not responsible for any error and/or omissions arising from translation and adaptation or otherwise.

Open access This is an open access article distributed in accordance with the Creative Commons Attribution Non Commercial (CC BY-NC 4.0) license, which permits others to distribute, remix, adapt, build upon this work non-commercially, and license their derivative works on different terms, provided the original work is properly cited, appropriate credit is given, any changes made indicated, and the use is non-commercial. See: http://creativecommons.org/licenses/by-nc/4.0/.

\section{ORCID iDs}

Rita McMorrow http://orcid.org/0000-0002-2835-9504

Barbara Hunter http://orcid.org/0000-0002-1268-3166

Jon Emery http://orcid.org/0000-0002-5274-6336

Jo-Anne Manski-Nankervis http://orcid.org/0000-0003-2153-3482

\section{REFERENCES}

1 Saeedi P, Petersohn I, Salpea P, et al. Global and regional diabetes prevalence estimates for 2019 and projections for 2030 and 2045: Results from the International Diabetes Federation Diabetes Atlas, $9^{\text {th }}$ edition. Diabetes Res Clin Pract 2019;157:107843.

2 Peyrot M, Rubin RR, Lauritzen T, et al. Psychosocial problems and barriers to improved diabetes management: results of the crossnational diabetes attitudes, wishes and needs (dawn) study. Diabet Med 2005;22:1379-85.

3 Nicolucci A, Kovacs Burns K, Holt RIG, et al. Correlates of psychological outcomes in people with diabetes: results from the second Diabetes Attitudes, Wishes and Needs (DAWN2 $\left({ }^{\mathrm{TM}}\right)$ ) study. Diabet Med 2016;33:1194-203.

4 Perrin NE, Davies MJ, Robertson N, et al. The prevalence of diabetes-specific emotional distress in people with type 2 diabetes: a systematic review and meta-analysis. Diabet Med 2017;34:1508-20.

5 Snoek FJ, Bremmer MA, Hermanns N. Constructs of depression and distress in diabetes: time for an appraisal. Lancet Diabetes Endocrinol 2015;3:450-60.

6 Skinner TC, Joensen L, Parkin T. Twenty-Five years of diabetes distress research. Diabet Med 2020;37:393-400.

7 Fisher L, Mullan JT, Skaff MM, et al. Predicting diabetes distress in patients with type 2 diabetes: a longitudinal study. Diabet Med 2009;26:622-7.

8 Pintaudi B, Lucisano G, Gentile S, et al. Correlates of diabetesrelated distress in type 2 diabetes: findings from the benchmarking network for clinical and humanistic outcomes in diabetes (BENCH-D) study. J Psychosom Res 2015;79:348-54.

9 Nanayakkara N, Pease A, Ranasinha S, et al. Depression and diabetes distress in adults with type 2 diabetes: results from the Australian National diabetes audit (ANDA) 2016. Sci Rep 2018;8:7846-46.

10 Goldney RD, Phillips PJ, Fisher LJ, et al. Diabetes, depression, and quality of life: a population study. Diabetes Care 2004;27:1066-70.

11 Hutter N, Schnurr A, Baumeister H. Healthcare costs in patients with diabetes mellitus and comorbid mental disorders--a systematic review. Diabetologia 2010;53:2470-9.

12 Gilmer TP, O'Connor PJ, Rush WA, et al. Predictors of health care costs in adults with diabetes. Diabetes Care 2005;28:59-64.

13 Park M, Katon WJ, Wolf FM. Depression and risk of mortality in individuals with diabetes: a meta-analysis and systematic review. Gen Hosp Psychiatry 2013;35:217-25.

14 Speight J, Hendrieckx C, Pouwer F, et al. Back to the future: 25 years of 'Guidelines for encouraging psychological well-being' among people affected by diabetes. Diabet Med 2020;37:1225-9.

15 Federation ID. Recommendations for managing type 2 diabetes in primary care, 2017.

16 Bradley C, Gamsu DS. Diabetes PW-bWGotWISVDAPf. In: Guidelines for encouraging psychological well-being. 1994: 11, 510-6.

17 Committee D, Canada D. Clinical practice guidelines for the prevention and management of diabetes in Canada. Can J Diabetes 2018;2018:42.

18 Garber AJ, Abrahamson MJ, Barzilay Jl, et al. Consensus statement by the American association of clinican endocrinologists and American college of endocrinology on the comprehensive type 2 diabetes management algorithm - 2019 executive summary. Endocr Pract 2019;25:69-100. 
19 Young-Hyman D, de Groot M, Hill-Briggs F, et al. Psychosocial care for people with diabetes: a position statement of the American diabetes association. Diabetes Care 2016;39:2126-40.

20 RACGP.. General practice management of type 2 diabetes: 2016-18. East Melbourne, Vic, 2016.

21 Dawson J, Doll H, Fitzpatrick R, et al. The routine use of patient reported outcome measures in healthcare settings. BMJ 2010;340:c186.

22 Nano J, Carinci F, Okunade O, et al. A standard set of personcentred outcomes for diabetes mellitus: results of an international and unified approach. Diabet Med 2020;37:2009-2018.

23 Nicolucci A, Kovacs Burns K, Holt RIG, et al. Diabetes attitudes, wishes and needs second study (DAWN2 ${ }^{\mathrm{TM}}$ ): cross-national benchmarking of diabetes-related psychosocial outcomes for people with diabetes. Diabet Med 2013;30:767-77.

24 Greenhalgh J, Dalkin S, Gooding K. Functionality and feedback: a realist synthesis of the collation interpretation and utilisation of patient-reported outcome measures data to improve patient care, 2017.

25 Byrne JL, Davies MJ, Willaing I, et al. Deficiencies in postgraduate training for healthcare professionals who provide diabetes education and support: results from the diabetes attitudes, wishes and needs (DAWN2) study. Diabet. Med. 2017;34:1074-83.

26 Hadjiconstantinou M, Dunkley AJ, Eborall H, et al. Perceptions of healthcare professionals and people with type 2 diabetes on emotional support: a qualitative study. BJGP Open 2020;4 doi:10.3399/bjgpopen20X101018. [Epub ahead of print: 01 May 2020].

27 Schmidt CB, van Loon BJP, Vergouwen ACM, et al. Systematic review and meta-analysis of psychological interventions in people with diabetes and elevated diabetes-distress. Diabet Med 2018. doi:10.1111/dme.13709. [Epub ahead of print: 13 Jun 2018].

28 Sturt J, Dennick K, Hessler D, et al. Effective interventions for reducing diabetes distress: systematic review and meta-analysis. Int Diabet Nurs 2015;12:40-55.

29 Chew BH, Vos RC, Metzendorf M-I, et al. Psychological interventions for diabetes-related distress in adults with type 2 diabetes mellitus. Cochrane Database Syst Rev 2017;9:Cd011469.

30 Boyce MB, Browne JP, Greenhalgh J. The experiences of professionals with using information from patient-reported outcome measures to improve the quality of healthcare: a systematic review of qualitative research. BMJ Qual Saf 2014;23:508-18.

31 Foster A, Croot L, Brazier J, et al. The facilitators and barriers to implementing patient reported outcome measures in organisations delivering health related services: a systematic review of reviews. $J$ Patient Rep Outcomes 2018;2:46.

32 Moher D, Shamseer L, Clarke M, et al. Preferred reporting items for systematic review and meta-analysis protocols (PRISMA-P) 2015 statement. Syst Rev 2015;4:1.

33 Kroenke K, Spitzer RL, Williams JBW. The PHQ-15: validity of a new measure for evaluating the severity of somatic symptoms. Psychosom Med 2002;64:258-66.

34 Radloff LS. The CES-D scale: a self-report depression scale for research in the general population. Appl Psychol Meas 1977;1:385-401.

35 Polonsky WH, Anderson BJ, Lohrer PA, et al. Assessment of diabetes-related distress. Diabetes Care 1995;18:754-60.

36 Polonsky WH, Fisher L, Earles J, et al. Assessing psychosocial distress in diabetes: development of the diabetes distress scale. Diabetes Care 2005;28:626-31.

37 Janssen MF, Pickard AS, Golicki D, et al. Measurement properties of the EQ-5D-5L compared to the EQ-5D-3L across eight patient groups: a multi-country study. Qual Life Res 2013;22:1717-27.

38 Brazier JE, Harper R, Jones NM, et al. Validating the SF-36 health survey questionnaire: new outcome measure for primary care. BMJ 1992;305:160-4.

39 Toobert DJ, Hampson SE, Glasgow RE. The summary of diabetes self-care activities measure: results from 7 studies and a revised scale. Diabetes Care 2000;23:943-50.

40 Covidence. Covidence systematic review software. veritas health innovation. Available: https://www.covidence.org [Accessed 17 Mar 2020].

41 The Cochrane Collaboration. Review manager web (RevMan web), 2019. Available: revman.cochrane.org

42 Higgins JPT, Altman DG, Gøtzsche PC, et al. The Cochrane collaboration's tool for assessing risk of bias in randomised trials. BMJ 2011;343:d5928.

43 Sterne JA, Hernán MA, Reeves BC, et al. ROBINS-I: a tool for assessing risk of bias in non-randomised studies of interventions. BMJ 2016;355:i4919.

44 Higgins JP, Thomas J, Chandler J. Cochrane handbook for systematic reviews of interventions. John Wiley \& Sons, 2019.

45 Schünemann HJ HJ, Vist GE, Glasziou P. Completing 'Summary of findings' tables and grading the certainty of the evidence. In: Cochrane handbook for systematic reviews of interventions version 60. Cochrane, 2019: 375-402

46 Ventura AD BJ, Holmes-Truscott E, Hendrieckx C. Diabetes MILES-2 2016 survey report. Melbourne: Diabetes Victoria, 2016. 